\title{
Very High Resolution Simulations of Compressible, Turbulent Flows
}

P. R. Woodward, D. H. Porter, I. Sytine, S. E. Anderson, A. A. Mirin, B. C. Curtis, R. H. Cohen, W. P. Dannevik, A. M. Dimits, D. E. Eliason, K.-H. Winkler, S. W. Hodson

This article was submitted to

$4^{\text {th }}$ Universidad Nacional Autonoma de Mexico Supercomputing Conference, Mexico City, Mexico, June 27-30, 2000

U.S. Department of Energy

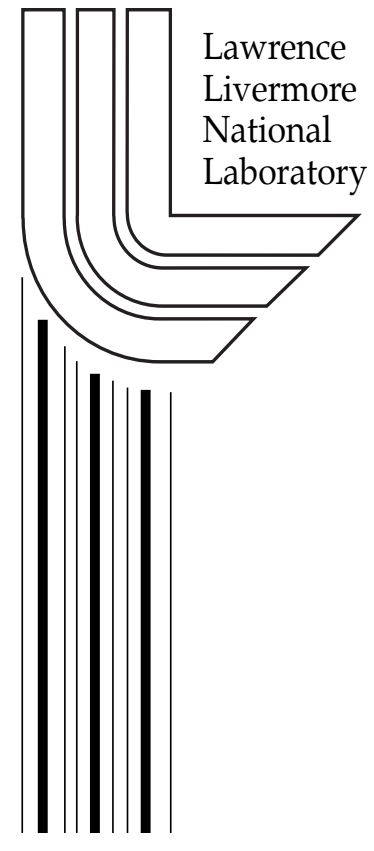




\section{DISCLAIMER}

This document was prepared as an account of work sponsored by an agency of the United States Government. Neither the United States Government nor the University of California nor any of their employees, makes any warranty, express or implied, or assumes any legal liability or responsibility for the accuracy, completeness, or usefulness of any information, apparatus, product, or process disclosed, or represents that its use would not infringe privately owned rights. Reference herein to any specific commercial product, process, or service by trade name, trademark, manufacturer, or otherwise, does not necessarily constitute or imply its endorsement, recommendation, or favoring by the United States Government or the University of California. The views and opinions of authors expressed herein do not necessarily state or reflect those of the United States Government or the University of California, and shall not be used for advertising or product endorsement purposes.

This is a preprint of a paper intended for publication in a journal or proceedings. Since changes may be made before publication, this preprint is made available with the understanding that it will not be cited or reproduced without the permission of the author.

This work was performed under the auspices of the United States Department of Energy by the University of California, Lawrence Livermore National Laboratory under contract No. W-7405-Eng-48.

This report has been reproduced directly from the best available copy.

Available electronically at http://www.doc.gov/bridge

Available for a processing fee to U.S. Department of Energy

And its contractors in paper from

U.S. Department of Energy

Office of Scientific and Technical Information

P.O. Box 62

Oak Ridge, TN 37831-0062

Telephone: (865) 576-8401

Facsimile: (865) 576-5728

E-mail: reports@adonis.osti.gov

Available for the sale to the public from

U.S. Department of Commerce

National Technical Information Service

5285 Port Royal Road

Springfield, VA 22161

Telephone: (800) 553-6847

Facsimile: (703) 605-6900

E-mail: orders@ntis.fedworld.gov

Online ordering: http://www.ntis.gov/ordering.htm

OR

Lawrence Livermore National Laboratory

Technical Information Department's Digital Library

http://www.llnl.gov/tid/Library.html 


\title{
VERY HIGH RESOLUTION SIMULATIONS OF COMPRESSIBLE, TURBULENT FLOWS
}

\author{
PAUL R. WOODWARD, DAVID H. PORTER, IGOR SYTINE, \\ S. E. ANDERSON \\ Laboratory for Computational Science \& Engineering \\ University of Minnesota, Minneapolis, Minnesota, USA \\ ARTHUR A. MIRIN, B. C. CURTIS, RONALD H. COHEN, WILLIAM P. DANNEVIK, \\ ANDRIS M. DIMITS, DONALD E. ELIASON \\ Lawrence Livermore National Laboratory \\ KARL-HEINZ WINKLER, STEPHEN W. HODSON \\ Los Alamos National Laboratory
}

\begin{abstract}
The steadily increasing power of supercomputing systems is enabling very high resolution simulations of compressible, turbulent flows in the high Reynolds number limit, which is of interest in astrophysics as well as in several other fluid dynamical applications. This paper discusses two such simulations, using grids of up to 8 billion cells. In each type of flow, convergence in a statistical sense is observed as the mesh is refined. The behavior of the convergent sequences indicates how a subgrid-scale model of turbulence could improve the treatment of these flows by high-resolution Euler schemes like PPM. The best resolved case, a simulation of a Richtmyer-Meshkov mixing layer in a shock tube experiment, also points the way toward such a subgrid-scale model. Analysis of the results of that simulation indicates a proportionality relationship between the energy transfer rate from large to small motions and the determinant of the deviatoric symmetric strain as well as the divergence of the velocity for the large-scale field.
\end{abstract}

\section{Introduction}

The dramatic improvements in supercomputing power of recent years are making possible simulations of fluid flows on grids of unprecedented size. The need for all this grid resolution is caused by the nearly universal phenomenon of fluid turbulence. Turbulence develops out of shear instabilities, convective instabilities, and Rayleigh-Taylor instabilities, as well as from shock interactions with any of these. In the tremendously high Reynolds number flows that are found in astrophysical situations, turbulence seems simply to be inevitable. Through the forward transfer of energy from large scale motions to small scale ones that characterizes fully developed turbulence, a fluid flow problem that might have seemed simple enough at first glance is made complex and difficult. Because the turbulent motions on small scales can strongly influence the large-scale flow, it is necessary to resolve the turbulence, at least to some reasonable extent, on the computational grid before the computed results converge (in a statistical sense). Thus it is that, more often than

Turbulent Flows submitted to World Scientific 4/22/2001 : 10:12 PM 
not, turbulence drives computational fluid dynamicists to refine their grids whenever increased computing power allows it. In this paper, we give examples of computations performed recently which illustrate where increased grid resolution is taking us. We focus our attention on the prospect that extremely highly resolved direct numerical simulations of turbulent flows can guide the development of statistical models for representing the effects of turbulent fluid motions that must remain unresolved in computations on smaller grids or of more complex problems.

\section{Homogeneous, Compressible Turbulence}

Perhaps the most classic example of a grid-hungry fluid dynamics problem is that of homogeneous, isotropic turbulence. Here we focus all available computational power on a small subdomain that could, in principle, have been extracted from of any of a large number of turbulent flows of interest. If from such a simulation we are able to learn the correct statistical properties of compressible turbulence, we can use our data to help test or construct appropriate subgrid-scale models of turbulent motions. When our computational grid is forced to contain an entire large-scale turbulent flow, we can then use such a model to make the computation practical. In the section that follows, we will see an example of such a larger flow in which we have used an 8-billion-cell grid in order to resolve both the large-scale flow and the turbulent fluid motions it sets up. In the results presented in this section, we will encounter signatures of the fully developed turbulence that can be recognized in a variety of such larger, more structured flows.

It is difficult to formulate boundary conditions that correctly represent those for a small subdomain of a larger turbulent flow. We use periodic boundary conditions here, but these are of course highly artificial, and therefore we must be careful not to over-interpret our results. Not only are our boundary conditions problematical, but our initial conditions also raise important issues. We rely on the theoretical expectation that, except for a small number of conserved quantities such as total energy, mass, and momentum, the details of our initial conditions will ultimately be "forgotten" as the turbulence develops, so that they will eventually become irrelevant. After a long time integration, we will find that the behavior of the flow on the scales comparable to the periodic length of our problem domain will remain influenced by both our initial conditions and by our periodic boundary conditions. However, the flow on shorter scales should be characteristic of fully developed turbulence. The flow on the very shortest scales, of course, must be affected by viscous dissipation and numerical discretization errors.

We are interested in the properties of compressible turbulence in the extremely high Reynolds number regime; we have no interest in the effects of viscosity, save upon the steady increase in the entropy of the fluid via the local dissipation of turbulent kinetic energy into heat. Therefore, we use an Euler method, PPM (the Piecewise-Parabolic Method [1-4,6]), in order to restrict the effects of viscous

Turbulent Flows submitted to World Scientific 4/22/2001: 10:12 PM 


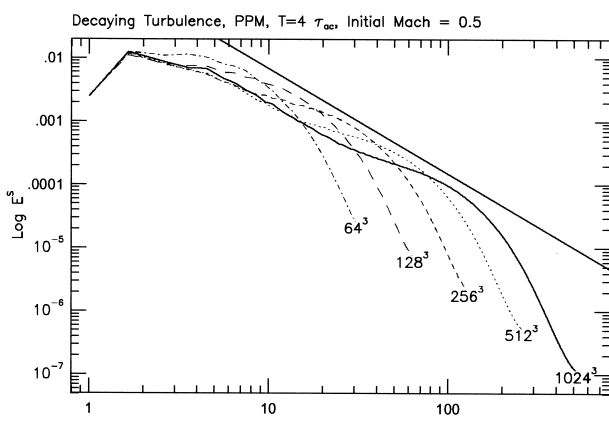

Figure 1: A comparison of velocity power spectra in 5 PPM runs on progresssively finer grids, culminating in a grid of a billion cells. All begin with identical initial states and all are shown at the same time, after the turbulence is fully developed. As the grid is refined, more and more of the spectrum converges to a common result. Spectra for the solenoidal (incompressible) component of velocity are shown in the top panel, while the spectra of the compressional component are at the bottom. The straight lines indicated in each panel show the Kolmogorov power law. Note

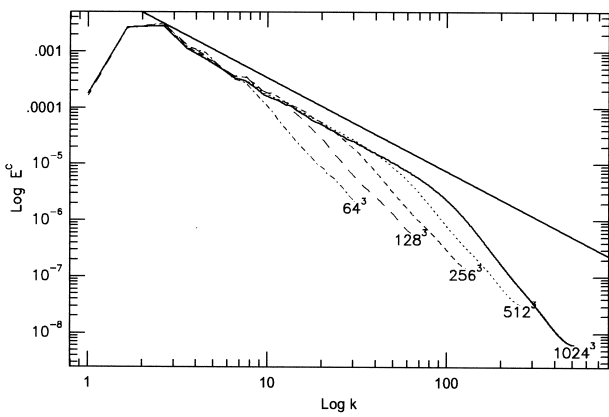

that agreement between the runs extends to considerably higher wavenumbers in the compressional spectra than in the solenoidal ones. This effect, first noted in our earlier work at $512^{3}$ grid resolution, was confirmed in the incompressible limit by simulations by Orszag and collaborators. A similar flattening of the power spectrum just above the dissipation scales has also been observed in data from experiments.

dissipation [9] to the smallest range of short length scales that we are able. A similar approach has been adopted by several other investigators [e.g. 15-18]. We must of course be careful to filter out the smallest-scale motions, which are affected by viscosity and other numerical errors, before we interpret our results as characterizing extremely high Reynolds number turbulence. This approach is in contrast to that adopted by many researchers, who attempt to approximate the behavior of flow in the limit of extremely high Reynolds numbers with the behavior of finite Reynolds number flows, where the Reynolds numbers are only thousands or less. In principle, an Euler computation gives an approximation to the limit of Navier-Stokes flows as the viscosity and thermal conductivity tend to zero. For our gas dynamics flows, this limit should be taken with a constant Prandtl number of unity. Much practical experience over decades of using Euler codes like PPM indicates that this intended convergence is actually realized. However, we must be aware that for turbulent flows, convergence, of course, occurs only in a statistical sense.

We can verify the convergence of our simulation results in this particular case in two ways. First, we can compare results from a series of simulations carried out on different grids. To demonstrate convergence, we look at the velocity power spectra, in Figure 1, obtained from these flow simulations. We expect that as the grid is successively refined, the velocity power spectrum on large scales does not

Turbulent Flows submitted to World Scientific 4/22/2001: 10:12 PM 

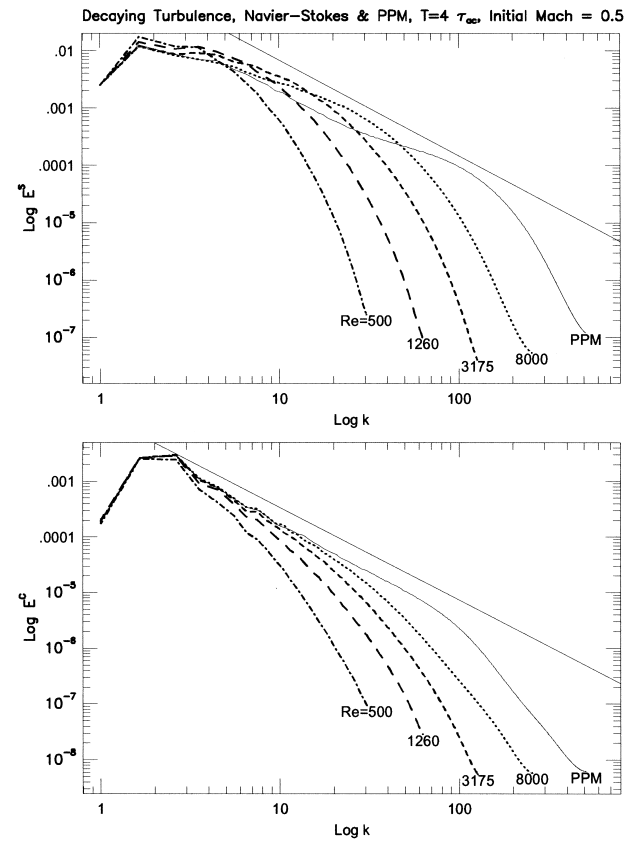

Figure 2: PPM Navier-Stokes simulations are here compared with the billion-cell PPM Euler simulation of Figure 1. Again, decaying compressible, homogeneous turbulence is being simulated on progressively finer grids of $64^{3}, 128^{3}, 256^{3}$, and $512^{3}$ cells. On each grid the smallest NovierStokes dissipation coefficients are used that are consistent with an accurate computation. Reynolds numbers are 500, 1260 , 3175,8000 . All runs begin with the same initial condition and are shown at the same time, after 4 sound crossings of the principal energy containing scale. As with the PPM Euler runs in Figure 1, this convergence study (to the infinite Reynolds number limit that we seek) shows that the compressional spectra, in the lower panel, are converged over a longer range in wavenumber than the solenoidal spectra at the top. In fact, the solenoidal spectra display no "inertial range," with Kolmogorov's $k^{-5 / 3}$ power law, at all. These results and those in Figure 1 indicate that the Euler spectra are accurate to about 4 times higher wavenumbers than NavierStokes.

change, while that on small scales is altered. The part of the spectrum that does not change on each successive grid refinement should, ideally, extend to twice the wavenumber each time the grid is refined. That this behavior is in fact observed is shown in Figure 1, taken from [8].

We would also like to verify that our procedure not only converges, but that it converges to the high Reynolds number limit of viscous flows. We can do this by comparing Navier-Stokes simulations of this same problem, carried out on a series of successively refined grids, with our PPM Euler simulation on the finest, billioncell grid. This comparison is shown in Figure 2, also taken from [8]. (A similar comparison for 2-D turbulence is given in [7].) The Navier-Stokes simulations do not achieve sufficiently high Reynolds numbers to unquestionably establish that they are converging to the same limit solution as the Euler runs. This is because the $512^{3}$ grid of the finest Navier-Stokes run has only a Reynolds number of 8000 . This Reynolds number has been limited by our demand that each Navier-Stokes simulation represent a run in which the velocity power spectrum has converged. This convergence has been checked, on the coarser grids where this can be done, by refining the grid while keeping the coefficients of viscosity and thermal conductivity constant and by verifying that the velocity power spectrum agrees over the entire range possible. We are confident that, had we been able to afford to carry the sequence of Navier-Stokes simulations forward to grids of $2048^{3}$ or $4096^{3}$ cells, we

Turbulent Flows submitted to World Scientific 4/22/2001 : 10:12 PM 
would have been able to obtain strict agreement with the already converged portion of the PPM velocity power spectrum on the billion-cell grid. At this time, such a demonstration is not practical.

We can use the detailed data from the billion-cell PPM turbulence simulation to test the efficacy of proposed subgrid-scale turbulence models. We can filter out the shortest scales affected either directly (from wavelengths of $2 \Delta \mathrm{x}$ to about $8 \Delta \mathrm{x}$ ) or indirectly (from about $8 \Delta \mathrm{x}$ to about $32 \Delta \mathrm{x}$ ) by the numerical dissipation of the PPM scheme. We are then left with the energy-containing modes, from wavelengths of about $256 \Delta \mathrm{x}$ to $1024 \Delta \mathrm{x}$, and the turbulent motions that these induce, from about $256 \Delta \mathrm{x}$ to about $32 \Delta \mathrm{x}$. A large eddy simulation, or LES, would involve direct numerical computation of these long wavelength disturbances with statistical "subgrid-scale" modeling of the turbulence. We can test a subgrid-scale turbulence model with this data by comparing the results it produces, in a statistical fashion, with those which our direct computation on the billion-cell grid has produced in the wavelength range between 256 and $32 \Delta x$. If we were to identify a subgrid-scale model that could perform an adequate job, as measured by the above procedure, then we should be able to add it to our PPM Euler scheme on the billion-cell grid. We will discuss aspects of such a model in the next section. Such a statistical turbulence model should, in the case of PPM, not be applied at the grid scale, as is generally advocated in the turbulence community, but instead at the scale where PPM's numerical viscosity begins to damp turbulent motions. This scale is about 8 $\Delta \mathrm{x}$, as can easily be verified by direct PPM simulation of individual eddies, as in [9], or by examination of the power spectra in Figure 1 (see especially the power spectra for the compressible modes).

In earlier articles (see for example [5]) we have suggested that the flattening of the velocity power spectra just before the dissipation range, seen in both PPM and Navier-Stokes simulations (see Figs. 1 and 2), is the result of diminished forward transfer of energy to smaller scales. This diminished forward energy transfer is caused by the lack of such smaller scales in the flow as a result of the action of the viscous dissipation. Applying an eddy viscosity from a statistical model of turbulence on scales around $8 \Delta x$ in a PPM turbulence simulation should, if the eddy viscosity has the proper strength, alleviate the above mentioned distortion of the forward energy transfer and make the simulated motions more correct in the range from $32 \Delta \mathrm{x}$ to $8 \Delta \mathrm{x}$, where we observe the flattening of the power spectra for the solenoidal modes in PPM Euler calculations. If the turbulence model, under the appropriate conditions, produces a negative viscous coefficient, this should help to give the PPM simulated flow the slight kick needed for it to develop turbulent motions on the scales resolved by the grid in this same region from about $32 \Delta \mathrm{x}$ to about $8 \Delta \mathrm{x}$. Thus we can hope that a successful statistical model of turbulence would make the computed results of such an LES computation with PPM accurate right down to the dissipation range at wavelengths of about $8 \Delta x$ for both the compressible and the solenoidal components of the velocity field. We note that

Turbulent Flows submitted to World Scientific 4/22/2001: 10:12 PM 

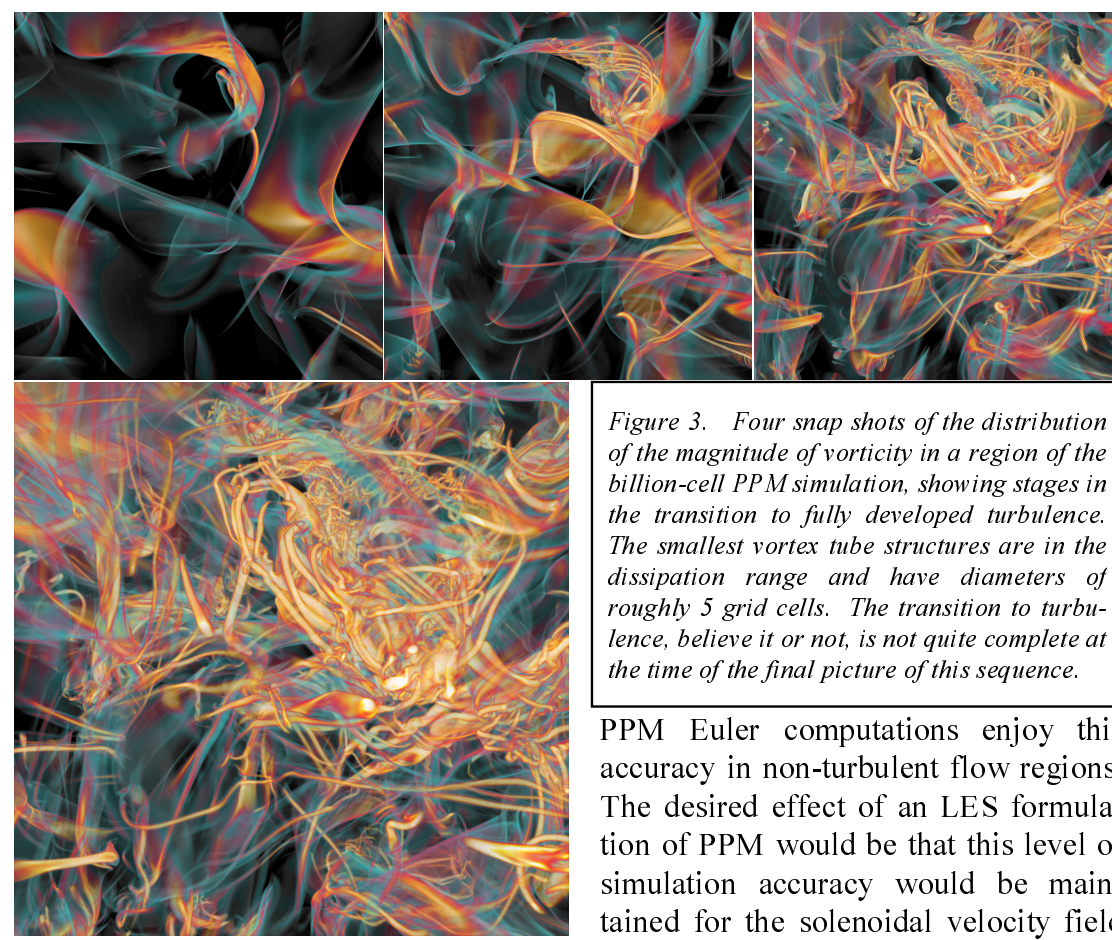

Figure 3. Four snap shots of the distribution of the magnitude of vorticity in a region of the billion-cell PPM simulation, showing stages in the transition to fully developed turbulence. The smallest vortex tube structures are in the dissipation range and have diameters of roughly 5 grid cells. The transition to turbulence, believe it or not, is not quite complete at the time of the final picture of this sequence.

PPM Euler computations enjoy this accuracy in non-turbulent flow regions. The desired effect of an LES formulation of PPM would be that this level of simulation accuracy would be maintained for the solenoidal velocity field within turbulent regions as well. Such an LES formulation should improve the ability of the numerical scheme to compute correct flow behavior, so that in these regions it would match the results of a PPM Euler computation on a grid refined by a factor between 2 and 4 in each spatial dimension and time. The enhanced resolving power in these regions of such a PPM LES scheme over an accurate Navier-Stokes simulation at the highest Reynolds number permitted by the grid would be very much greater still, as the power spectra in Figure 2 clearly indicate. In this statement, we have of course assumed that an approximation to the infinite Reynolds number limit is desired, and not a simulation of the flow at any attainable finite Reynolds number. In astrophysical calculations, as in many other circumstances, this is generally the case.

In addition to providing the essential, highly resolved simulation data needed to validate subgrid-scale turbulence models for use in our PPM scheme, our billion-cell PPM Euler simulation of homogeneous, compressible turbulence gives a fascinating glimpse at the process of transition to fully developed turbulence. In the sequence of snap shots of the distribution of the magnitude of vorticity in this flow given in Figure 3, we see that the vortex sheet structures that emerged from our random stirring of the flow on very large scales develop concentrations of vorticity in ropes

Turbulent Flows submitted to World Scientific 4/22/2001 : 10:12 PM 
that become vortex tubes. These vortex tubes in turn entwine about each other as the flow becomes entirely turbulent. We note that our first billion-cell simulation of this type was performed in collaboration with Silicon Graphics, who in 1993 built a prototype cluster of multiprocessor machines expressly for attacking such very large computational challenges. The simulation shown in Figure 3 was performed in 1997 on a cluster of Origin 2000 machines from SGI at the Los Alamos National Laboratory. Although we have used results of this simulation to test subgrid-scale turbulence model concepts, we will discuss detailed ideas for such models in the context of an even more highly resolved flow.

\section{Turbulent Fluid Mixing at an Unstably Accelerated Interface}

An example of a turbulent flow driven by a large-scale physical mechanism is that of the unstable shock-acceleration of a contact discontinuity (a sudden jump in gas density) in a gas. This calculation was carried out as part of the DoE ASCI program's verification and validation activity, and it was intended to simulate a shock tube experiment of Vetter and Sturtevant (1995) at Caltech [10]. In the laboratory experiment, air and sulfur hexaflouride were separated by a membrane in a shock tube, and a Mach 1.5 shock impinged upon this membrane, forcing it through an adjacent wire mesh and rupturing it. The interface between the two gases is unstable when accelerated by a shock, and both the large-scale flexing of the membrane and the wire mesh impart perturbations that are amplified by this Richtmyer-Meshkov instability. The conditions of this problem therefore provide a context to observe the competition and interaction of small- and large-scale perturbations of the interface along with the turbulence that develops.

In Figure 4, at the top left on the next page, a thin slice through the unstable mixing region between the two gases is shown in a volume rendering of the entropy of the gas. The entropy, after the initial shock passage, is a constant of the motion, with different values in each gas. In the figure, white corresponds to the entropy of the pure initially denser gas, while the pure initially more diffuse gas is made transparent. The regions of intermediate colors in the figure show different proportions of mixing of the two fluids within the individual cells of the $1920 \times 2048^{2}$ grid ( 8 billion cells). Below this image, a volume rendering of the enstrophy, the square of the vorticity, is shown for the same slice. This simulation was carried out on the Lawrence Livermore National Laboratory's large ASCI IBM SP system using 3904 CPUs. A constraint of this particular supercomputing opportunity was that the previously tuned simplified version, sPPM [6], of our PPM [1-4] gas dynamics code had to be used. This constraint limited us to a single-fluid model with a single gamma-law equation of state to simulate the air and sulfur hexaflouride system in the Caltech experiment. We chose $\gamma=1.3$ and initial densities of 1.0 and 4.88 to represent the experiment as best we could under these constraints. The interface perturbation was $0.01 \times[-\cos (2 \pi x) \cos (2 \pi y)+|\sin (10 \pi x) \sin (10 \pi y)|]$. We initialized the fluid interface as a smooth transition spread over a width of 5 grid

Turbulent Flows submitted to World Scientific 4/22/2001: 10:12 PM 

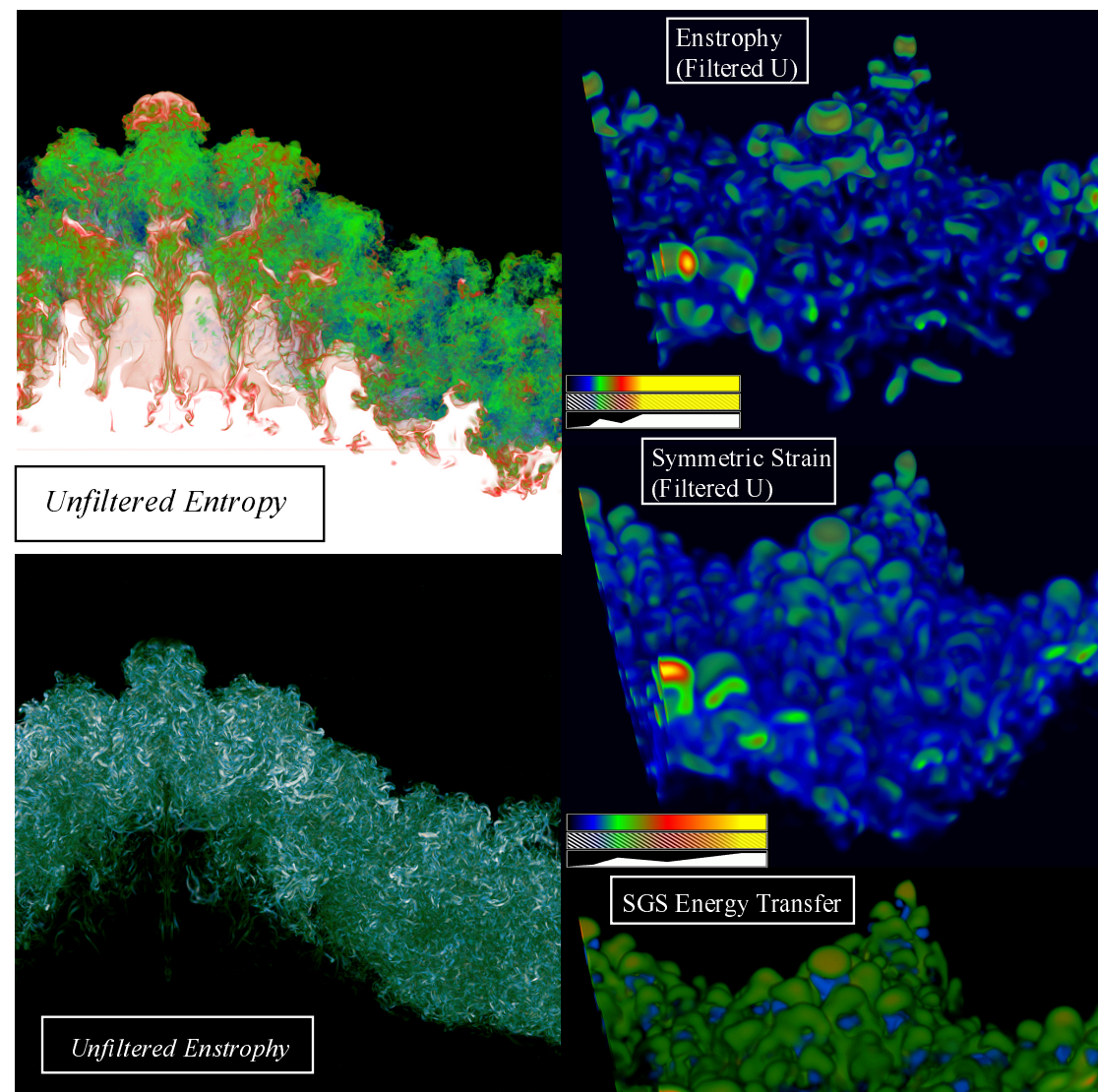

cells. This initialization greatly reduced the amplitude of the high frequency signals that are unavoidable in any gridbased method. The sPPM method of capturing and advecting fluid interfaces forces smearing of these transitions over about 2 grid cells and resists, through its inherent numerical diffusion, development of very short wavelength perturbations. By setting up the initial interface so smoothly, we assured that after its shock compression it would contain only short wavelength perturbations that the SPPM scheme was designed to handle. Nevertheless, the flow is unstable, so one must be careful in interpreting the results.

A detailed discussion of the results of this simulation will be presented elsewhere [11]. As with the homogeneous turbulence simulations discussed earlier and our simulations of compressible convection in stars [9, 12-14], this Richtmyer-

Turbulent Flows submitted to World Scientific 4/22/2001 : 10:12 PM 
Meshkov problem demonstrates convergence upon mesh refinement to a velocity power spectrum, in this case for the longitudinal velocity, shown in the figure at the top right on the next page, that demonstrates an energycontaining range determined by the initial and boundary conditions of the problem with a short inertial range with Kolmogorov $k^{-5 / 3}$ scaling. At the highest wavenumbers, the numerical dissipation of the SPPM Euler scheme is at work, and there is again, just above

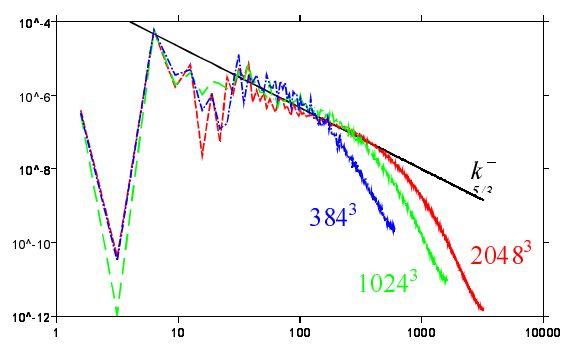

Power spectra for the longitudinal velocity at the midplane in 3 Richtmyer-Meshkov simulations with sP PM on progressively finer grids.

this dissipation range, a short segment of the spectrum with a slope flatter than the Kolmogorov trend. The 8-billion-cell grid of this calculation is so fine that it allows us to apply a Gaussian filter with full width at half maximum of 67.8 cells, producing a complex filtered flow consisting mostly of the energy-containing modes (a 128-cell sine wave is damped by a factor of $1 / e$ ). We interpret the many well-resolved modes at scales removed by the filter as fully developed turbulence. Using the PPM code, we might hope to capture the modes of the filtered fields on a grid of $256^{3}$ cells. If we can use our 8-billion-cell data to characterize the statistical effects of the turbulent modes beneath the 128-cell scales preserved by the filter, then an LES calculation on a very much coarser grid that made use of this characterization might succeed in producing the correct statistically averaged behavior of the mixing layer. With this goal in mind, we consider the rate, $F_{S G S}$, of forward energy transfer from the modes preserved by the filter to those eliminated by it.

If we denote the filtered value of a variable, such as the density $\rho$, by an overbar, $\bar{\rho}$, then we will denote by a tilde the result of a mass-weighted filtering, as for the velocity: $\tilde{u}=\overline{\rho u} / \bar{\rho}$. With these definitions, the filter when applied to the equation of momentum conservation produces the following result:

$$
\frac{\partial \overrightarrow{\rho u_{i}}}{\partial t}+\partial_{j}\left(\bar{\rho} \tilde{u}_{i} \tilde{u}_{j}\right)=-\partial_{i} \bar{p}-\partial_{j} \tau_{i j}
$$

where $\tau_{i j}=\overline{\rho u_{i} u_{j}}-\bar{\rho} \widetilde{u}_{i} \widetilde{u}_{j}$ is a quantity that we call the "subgrid-scale" stress (considering, for our present purposes, the computed structures below the filter scale to be "subgrid-scale" structures in an imagined LES computation). The filtered kinetic energy is now $\widetilde{K}=\bar{\rho} \widetilde{u}^{2} / 2$, and the equation for $\partial \widetilde{K} / \partial t$ that can be derived from the momentum equation above and the continuity equation contains a term $-\tilde{u}_{i} \partial_{j} \tau_{i j}$, which is in turn $-\partial_{j}\left(\tilde{u}_{i} \tau_{i j}\right)+\left(\partial_{j} \tilde{u}_{i}\right) \tau_{i j}$. The first term in this second expression is the divergence of an energy flux, and the negative of the

Turbulent Flows submitted to World Scientific 4/22/2001 : 10:12 PM 
second term, $-\left(\partial_{j} \widetilde{u}_{i}\right) \tau_{i j}$, we identify as the rate of kinetic energy transfer, $F_{S G S}$, from the modes on scales larger than our filter to the "subgrid-scale" modes on smaller scales. In the illustrations two pages earlier, this forward energy transfer rate, $F_{S G S}$, is visualized along with the enstrophy (square of the vorticity for $\tilde{u}$ ) and the deviatoric symmetric strain, $\quad \Pi^{2}=\sum_{i j}\left(\frac{\partial \tilde{u}_{i}}{\partial x_{j}}+\frac{\partial \tilde{u}_{j}}{\partial x_{i}}-\frac{2}{3} \delta_{i j} \nabla \cdot \vec{u}\right)^{2}$.

Although there is a positive correlation between $F_{S G S}$ and $\Pi^{2}$, it is clear from these images that the forward energy transfer rate is both positive and negative. Thus if we use $\Pi^{2}$ to model $F_{S G S}$, we must include another factor which switches sign at the appropriate places in the flow. The situation is clearest in the region of the largest plume in the center of the problem domain. The "mushroom cap" at the top of this plume is essentially a large ring vortex, as indicated in the diagram at the lower right. Near the top of the plume, the top in the diagram, there is an approximate stagnation flow, with compression in the direction along the plume and with expansion in the two dimensions of the plane perpendicular to this, the plane of the original unstable layer. The transfer of energy to small scales is large in this region. Here we believe that small-scale line vortices are stretched so that they tend to become aligned in this plane in myriad directions, which leads to their mutual disruption to produce even smaller-scale line vortex structures. We have observed this process in great detail at the tops of rising, buoyant plumes in our earlier highresolution simulations of stellar convection (cf. $[13,14]$ and particularly the movie looking down on the top of the simulated convective layer). At the base of the vortex ring in the diagram at the right, and in the lower portion of the "mushroom cap" of the large, central plume in the simulated Richtmyer-Meshkov mixing layer, the flow compresses in two dimensions while it expands in the third, the dimension along the length of the plume. Here there is energy transfer from the small scales to the large, as indicated by negative values of $F_{S G S}$ in the volume-rendered image. Here we believe that vortex tubes become aligned in the single stretching direction, so that they are likely to interact by entwining themselves around each other to form larger vortex tube structures, a process that we have also observed in greater detail in our stellar convection studies (cf. $[13,14]$ and the portion of the movie that shows the conglomeration of vertically aligned vortex tubes in the downflow lanes along the edges of the convection cells). This behavior of the forward energy

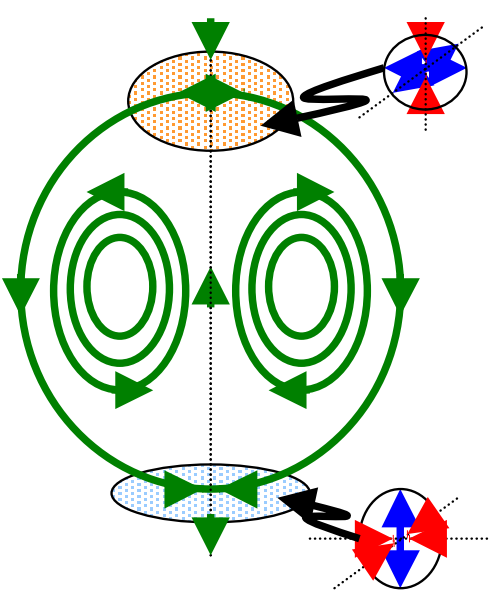

Turbulent Flows submitted to World Scientific 4/22/2001 : 10:12 PM $10 / 13$ 
transfer rate, $F_{S G S}$, with its sign dependence on the nature of the local flow field, gives us the hint that this transfer rate, which must be central to any successful subgrid-scale model of turbulence, should be modeled in terms of the determinant, $\operatorname{det}(\Pi)$, of the deviatoric symmetric strain tensor for the filtered velocity field, which flips sign in the appropriate locations (since the determinant is just the product of the 3 eigenvalues of the matrix). This yields a much better correlation than using $\Pi^{2}$, as is the more usual choice.

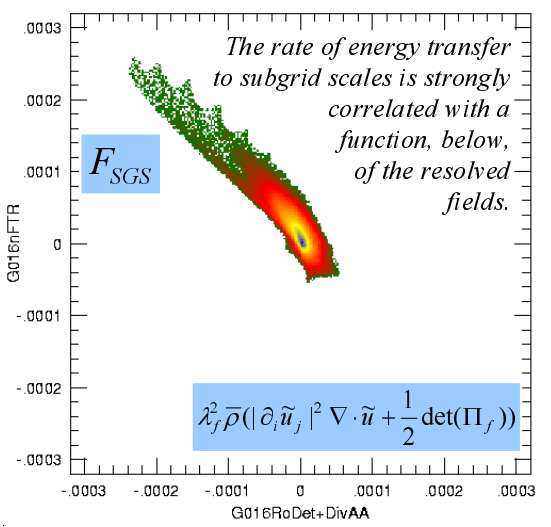
The correlation is improved still further by including the obvious dependence of $F_{S G S}$ on the divergence of the filtered velocity field, which transports turbulent kinetic energy from larger to smaller scales by simply compressing the overall flow in a region. Thus we obtain:

$$
F_{S G S} \propto-\lambda_{f}^{2} \bar{\rho}\left(\left|\partial_{i} \tilde{u}_{j}\right|^{2} \nabla \cdot \tilde{u}+\frac{1}{2} \operatorname{det} \Pi_{f}\right)
$$

where $\Pi_{f}$ represents $\Pi$ for the filtered velocity field, and $\lambda_{f}$ is a filter wavelength, equal to 128 cells for our Gaussian filter with full width at half maximum of 67.8 cells. This correlation, which is excellent, is shown in the figure at the top right. The data from our billion-cell simulation of homogeneous, compressible turbulence, described in the first part of this article, supports this same model for $F_{S G S}$ with an equally strong correlation to that shown in the figure here, even though in that flow the divergence of the filtered velocity field, with an rms Mach number of about 1/3, tends to dominate the term in $\Pi_{f}$ on the right in the above relationship. For this data, as described earlier, two filters are used, with full widths at half maximum of 67.8 and 6.03 cells.

The relation for $F_{S G S}$ given above can perhaps be used in building a $k-\varepsilon$ model of subgrid-scale turbulence. In this case, a model for the subgrid-scale stress, $\tau_{i j}$, that produces this relation for $F_{S G S}$ is $\tau_{i j}=k \delta_{i j}+A \Pi_{i j}$, where $k$, the subgrid-scale turbulent kinetic energy, $\tau_{i i}$, is approximated by $\lambda_{f}^{2} \bar{\rho}\left|\partial_{i} \tilde{u}_{j}\right|^{2}$ and where $A=\left(\lambda_{f}^{2} \bar{\rho} / 2\right)\left(\operatorname{det}(\Pi) /|\Pi|^{2}\right)$ can be either positive or negative. This suggests a mechanism for incorporating the model into the momentum and total energy conservation laws of a numerical scheme such as PPM while maintaining the scheme's strict conservation form. The approximation of $k$, the subgrid-scale turbulent kinetic energy, involving velocity changes on the scale of the filter demands that, in proper LES style, the larger turbulent eddies are resolved on the

Turbulent Flows submitted to World Scientific 4/22/2001 : 10:12 PM $11 / 13$ 
grid, so that these velocity changes are meaningful. In the spirit of a $k$ - $\varepsilon$ model, we could replace $\lambda_{f}^{2} \bar{\rho}\left|\partial_{i} \widetilde{u}_{j}\right|^{2}$ by $k$ in the relation for $F_{S G S}$ and use the resulting form for $F_{S G S}$ as the time rate of change of $k$ in a frame moving with the velocities $\tilde{u}_{j}$, that is, with the resolved velocity field in the LES calculation. A further term, related to the $\varepsilon$ of a $\mathrm{k}-\varepsilon$ model, representing the decay of $k$ due to viscous dissipation on scales well below that of the grid would also have to be included. We would then have a dynamical partial differential equation for $k$ to solve along with the conservation laws for mass, momentum, and total energy. Constructing such a subgrid-scale model of turbulence for use with the PPM gas dynamics scheme is a subject of future work.

\section{Acknowledgements}

We would like to acknowledge generous support for this work from the Department of Energy, through grants DE-FG02-87ER25035 and DE-FG02-94ER25207, contracts from Livermore and Los Alamos, and an ASCI VIEWS contract through NCSA; from the National Science Foundation, through its PACI program at NCSA, through a research infrastructure grant, CDA-950297, and also through a Grand Challenge Application Group award, ASC-9217394, through a subcontract from the University of Colorado; and from NASA, through a Grand Challenge team award, NCCS-5-151, through a subcontract from the University of Chicago. We would also like to acknowledge local support from the University of Minnesota's Minnesota Supercomputing Institute. This work was performed in part under the auspices of the U.S. DoE by the Lawrence Livermore National Laboratory under contract NO. W-7405-ENG-48.

\section{References}

1. Woodward, P. R., and Colella, P., 1984. "The Numerical Simulation of TwoDimensional Fluid Flow with Strong Shocks," J. Comput. Phys. 54, 115-173.

2. Colella, P., and Woodward, P. R., 1984. "The Piecewise-Parabolic Method (PPM) for Gas Dynamical Simulations," J. Comput. Phys. 54, 174-201.

3. Woodward, P. R., 1986. "Numerical Methods for Astrophysicists," in Astrophysical Radiation Hydrodynamics, eds. K.-H.Winkler and M. L. Norman, Reidel, 1986, pp. 245-326.

4. Edgar, B. K., Woodward, P. R., Anderson, S. E., Porter, D. H., and Dai, Wenlong, 1999. PPMLIB home page at http://www.lcse.umn.edu/PPMlib.

5. Porter, D. H., Woodward, P. R., and Pouquet, A., 1998. "Inertial Range Structures in Decaying Turbulent Flows," Physics of Fluids 10, 237-245.

Turbulent Flows submitted to World Scientific 4/22/2001 : 10:12 PM $12 / 13$ 
6. Woodward, P. R., and Anderson, S. E., 1995. SPPM benchmark code, LCSE version. Available at http://www.lcse.umn.edu.

7. Porter, D. H., Pouquet, A., and Woodward, P. R., 1992. "A Numerical Study of Supersonic Homogeneous Turbulence," Theoretical and Computational Fluid Dynamics, 4, 13-49.

8. Sytine, I. V., Porter, D. H., Woodward, P. R., Hodson, S. W., and Winkler, K.H., 2000. "Convergence Tests for Piecewise Parabolic Method and NavierStokes Solutions for Homogeneous Compressible Turbulence," J. Comput. Phys., 158, 225-238.

9. D. H. Porter and P. R. Woodward, "High Resolution Simulations of Compressible Convection with the Piecewise-Parabolic Method (PPM)," Astrophysical Journal Supplement, 93, 309-349 (1994).

10. Vetter, M., and Sturtevant, B., 1995. "Experiments on the Richtmyer-Meshkov Instability of an Air/SF6 Interface," Shock Waves, 4, 247-252.

11. R. H. Cohen, W. P. Dannevik, A. M. Dimits, D. E. Eliason, A. A. Mirin, Y. K. Zhou, D. H. Porter, and P. R. Woodward, "Three-Dimensional Simulation of a Richtmyer-Meshkov Instability with a Two-Scale Initial Perturbation," in preparation.

12. D. H. Porter, P. R. Woodward, and M. L. Jacobs, "Convection in Slab and Spheroidal Geometries," Proc. $14^{\text {th }}$ International Florida Workshop in Nonlinear Astronomy and Physics: Astrophysical Turbulence and Convection, Univ. of Florida, Feb., 1999; in Annals of the New York Academy of Sciences 898, 1-20 (2000). Available at http://www.lcse.umn.edu/convsph .

13. D. H. Porter and P. R. Woodward, "3-D Simulations of Turbulent Compressible Convection," Astrophysical Journal Supplement, 127, 159-187 (2000).

14. http://www.lcse.umn.edu/Movies - LCSE web site to visit in order to view movies of the simulations discussed in this article. See also other links at http://www.lcse.umn.edu, including subdirectories research/lanlrun, research/nsf, research/nsf/penconv96dec.html, research/RedGiant. A PDF version of this article, with high-quality color illustrations, can be found at this Web site, along with animations from the computer simulations discussed here.

15. http://www.llnl.gov/casc/asciturb - site for ASCI turbulence team at LLNL.

16. J. P. Boris, F. F. Grinstein, E. S. Oran, and R. J. Kolbe, "New Insights into Large Eddy Simulation," Fluid Dyn. Res. 19, 19 (1992).

17. C. Fureby, and F. F. Grinstein, "Monotonically integrated large eddy simulation of free shear flows," AIAA J. 37(5), 544 (1999).

18. G.-S. Karamanos and G. E. Karniadakis, "A Spectral Vanishing Viscosity Method for Large-Eddy Simulations," J. Comput. Phys. 163, 22-50 (2000).

Turbulent Flows submitted to World Scientific 4/22/2001 : 10:12 PM 